\title{
Elbow pain proven to be tuberculous arthritis -A case report-
}

\author{
Sun Soph Jung, Mi Kyoung Lee, Sang Ho Lim, Yong-Man Kwon, and Sang-Sik Choi \\ Department of Anesthesiology and Pain Medicine, Korea University Guro Hospital, Seoul, Korea
}

Elbow tuberculosis is a rare disease which accounts for $1-3 \%$ of all cases of osteoarticular tuberculosis. The diagnosis of tuberculous arthritis is very difficult in most clinical situations because of the insidious onset, indolent process, and mild and non-specific local or systemic symptoms. Thus, the confirmatory diagnosis and effective treatment for tuberculous arthritis are delayed until the disease progresses to an advanced stage in many clinical settings. Herein we report a case of septic arthritis of the elbow due to Mycobacterium tuberculosis as a result of misdiagnosis and delayed treatments. (Korean J Anesthesiol 2010; 58: 211-214)

Key Words: Elbow tuberculosis, Tuberculous arthritis.

Musculoskeletal tuberculosis is a very rare disease entity, and it accounts for $1-5 \%$ of total tuberculous infections [1-5]. Tuberculous arthritis commonly occurs in the joints where weight loading is exerted or joints which are exposed to repeated episodes of trauma. The common sites of occurrence include the vertebral joints, which is the most prevalent site and accounts for approximately $50 \%$ of the total sites of involvement $[5,6]$, followed by the hip and knee joints $[2,3]$. Tuberculous arthritis occurring in the upper extremities is rare, accountings for $1-5 \%$ of musculoskeletal tuberculosis $[1,3,7]$. Due to an unclear manifestation of the symptoms, and despite substantial disease progression, an appropriate test or assessments are often not performed. Tuberculous arthritis is often misdiagnosed as degenerative arthritis or rheumatoid arthritis, an abnormality of tendons or ligaments, and diseases of connective tissues, including bursitis, around the joint [6]. As described herein, in cases in which a diagnosis of tuberculous arthritis was delayed or was misdiagnosed, it was mostly detected when the diseases progressed. Severe complications due to diseases might also occur. In the current case, treatment was performed based on suspected diagnoses of lateral epicondylitis and rheumatoid arthritis. Because the patient was refractory to treatment, however, a diagnosis of septic arthritis was made in light of the tuberculosis. Here, we report our case with a review of the literature.

\section{Case Report}

A 50-year-old woman sought evaluation in our clinic with a complaint of pain in the right elbow joint. Six months earlier, the

Received: September 25, 2009. Revised: 1st, October 14, 2009; 2nd, October 15, 2009. Accepted: October 19, 2009

Corresponding author: Sang-Sik Choi, M.D., Department of Anesthesiology and Pain Medicine, Korea University Guro Hospital, Guro-2dong, Guro-gu, Seoul 152-703, Korea. Tel: 82-2-818-6207, Fax: 82-2-851-9897, E-mail: clonidine@empal.com

(c)This is an open-access article distributed under the terms of the Creative Commons Attribution Non-Commercial License (http:// creativecommons.org/licenses/by-nc/3.0/), which permits unrestricted non-commercial use, distribution, and reproduction in any medium, provided the original work is properly cited. 
patient had pain in the right elbow and tenderness in the lateral epicondyle, so the patient received a trigger point injection (TPI) two times and intramuscular stimulation (IMS) under the impression of lateral epicondylitis. Following this treatments, the symptoms improved. The patient also received an anterior cervical discectomy and fusion 4 weeks prior to the outpatient visit, due to pain in the right neck and shoulder originating from an intervertebral disc herniation at C6/7. There was no history of trauma or underlying diseases.

At the time of the outpatient visit, a physical examination revealed tenderness in the lateral epicondyle and a positive finding on Cozen's test. In a systemic review, there were no respiratory symptoms, such as cough, sputum, and dyspnea or systemic symptoms, such as fever, chills, and weight loss.

In our patient, a recurrence of the lateral epicondylitis was presumed. A TPI, IMS, and physical therapy were administered again, which led to an improvement in symptoms. Despite the improvement in symptoms, however, on physical examination there was a persistent presence of findings, such as tenderness in the lateral epicondyle, limitations in the extension and flexion of the elbow joint, and limitations in the extension of the shoulder joint. Therefore, TPI and IMS were repeated 3 additional times for 3 weeks. The treatment response, however, was not typical. To make a differential diagnosis, a plain radiograph of the elbow joint and a hematologic examination were performed.

On plain radiography, there were no notable findings (Fig. 1). On the hematologic testing, the erythrocyte sedimentation rate (ESR) and C-reactive protein (CRP) were elevated to $100 \mathrm{~mm} / \mathrm{hr}$ and $25.5 \mathrm{mg} / \mathrm{L}$, respectively. The WBC count, rheumatoid factor

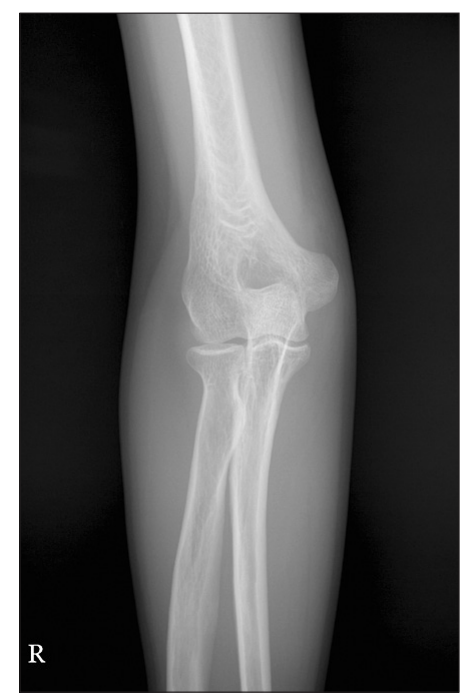

Fig. 1. Anteroposterior X-ray of the right elbow shows no significant abnormalities.
(RF), and fluorescent anti-nuclear antibody (FANA) were all normal.

We assumed that the elevated level of ESR and CRP represented seronegative rheumatoid arthritis, or less probable, nonspecific findings following the cervical herniation of nucleus pulposus (HNP) procedure. We then administered drug treatments using meloxicam ( $15 \mathrm{mg} /$ day) and prednisolone (10 mg/day). Following the initiation of drug treatments, the symptoms, such as the limitation in range of motion (ROM), were greatly improved. Approximately 1 month later, the patient voluntarily discontinued the drug treatment, and had an abrupt aggravation of symptoms. Severe pain arose during ambulation as well as during rest. Especially during the night, the pain was further aggravated. On physical examination, she had tenderness in the medial and lateral epicondyles. During extension of the elbow joint, there was a restriction in the motor area which was further aggravated.

When meloxicam (15 mg/day) and prednisolone (20 mg/day) were re-administered, the symptoms improved. Three weeks after the drug treatments resumed, methotrexate $(2.5 \mathrm{mg} /$ week $)$ and sulfasalazine ( $1 \mathrm{~g} /$ day) were added. One month later, the steroid treatment was discontinued. The drug treatment was performed for a total of 7 weeks. Thereafter, however, no followup observations were made.

Three months later, the symptoms became severe and the patient re-visited the clinic. On physical examination, there was severe limitation in elbow flexion and extension, and local warmth and swelling in the right elbow. Under the

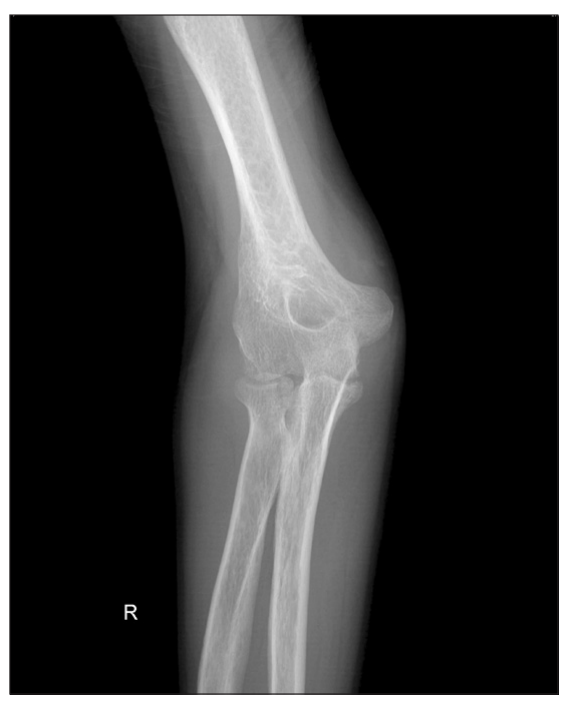

Fig. 2. Anteroposterior X-ray of the right elbow shows diffuse joint space narrowing, multiple marginal and central bone erosions, a significant amount of joint effusion, and slight periarticular osteoporosis. 


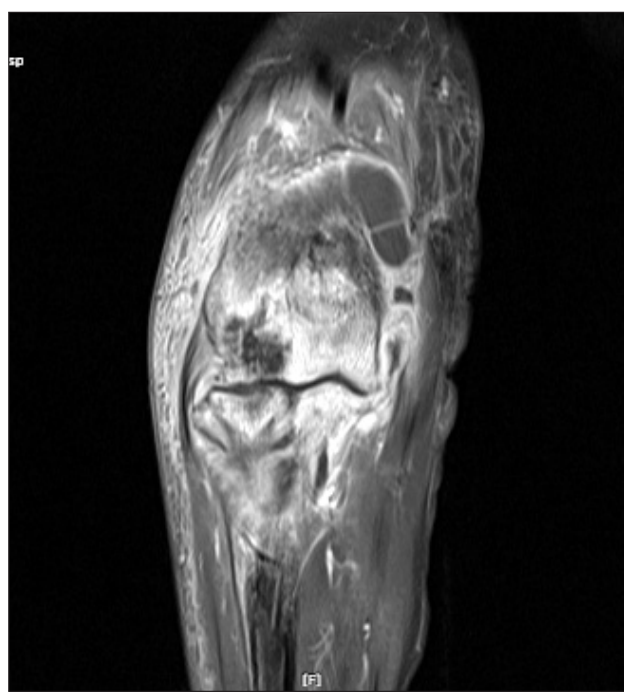

Fig. 3. A coronal enhanced T1-weighted MRI image shows diffuse joint space narrowing with severe erosive bone changes, and osteomyelitis of the distal humerus, proximal radius and ulna, myositis, and cellulitis of the muscle and subcutaneous layer around the elbow joint, and a large amount of joint effusion with synovial enhancement.

impression of septic lateral epicondylitis, a plain radiograph and magnetic resonance imaging (MRI) were performed. On the plain radiograph, there was a massive intra-articular effusion, multiple bone erosions around the joint, osteopenia, and an overall decrease in the articular cavity (Fig. 2). On MRI scans, there were also findings, such as osteomyelitis, involing the distal part of the radius, ulna, or humerus, periarticular myositis, and subcutaneous cellulitis (Fig. 3). Following an aspiration of the intra-articular effusion, the pus-like synovial fluid was aspirated. This led to the diagnosis of septic arthritis, which was followed by synovectomy and massive irrigation in an emergency setting.

The tuberculosis polymerase chain reaction (PCR) of the synovial fluid, there were positive findings of M. tuberculosis. A tissue biopsy of the synovial membrane or synovium was suggestive of a chronic caseating granulomatous inflammation, consistent with tuberculosis. Acid-fast bacilli (AFB) were identified

on specimen staining. For the treatment of tuberculosis following surgery, four-agent drug treatments were performed using isoniazid, rifampin, pyrazinamide, and ethambutol. At present, the patient's pain is greatly diminished. To maintain the ROM of the joint, the patient is receiving physical therapy.

\section{Discussion}

Musculoskeletal tuberculosis is a rare disease entity, accounting for $1-5 \%$ of tuberculous infections. Tuberculous arthritis occurring in the elbow joint is rare, and accounts for $1-5 \%$ of musculoskeletal tuberculosis cases.

Similar to others infectious arthritides, tuberculous arthritis affects a single joint. In $10 \%$ of cases, however, more than one joint can be affected. Musculoskeletal involvement is caused by hematogenous, lymphatic, or direct local spread of tubercle bacilli from other lesions, such as a quiescent pulmonary primary or another extraosseous focus $[3,7]$.

Symptoms of tuberculous arthritis are both unclear and obscure, which are clinically manifested as local swelling and chronic pain of a single joint accompanied by gradually decreased ROM. Joint swelling and pain exacerbated by activity are the most common manifestation [4].

Because of the indolent disease process and the subtle nature of symptoms, diagnostic evaluations are often not undertaken until the disease has progressed.

In the early stage of diseases, tuberculous arthritis may be easily mistaken for trauma or rheumatoid arthritis and septic arthritis. A significant delay between the onset of symptoms and the definitive diagnosis commonly ranges from 5-47 months [6]. Systemic symptoms may include a low fever, fatigue, decreased appetite, decreased weight, night chills, tachycardia, and anemia, but these symptoms are not common.

In the case presented herein, the patient complained of the pain present in the right elbow joint. Under the impression of lateral epicondylitis, the treatment was initiated, however, the patient had an atypical responses to these treatments and then underwent additional laboratory tests. This showed that such parameters as ESR and CRP were elevated to $100 \mathrm{~mm} / \mathrm{hr}$ and $25.5 \mathrm{mg} / \mathrm{L}$.

Based on the elevation of the ESR and CRP to $100 \mathrm{~mm} / \mathrm{hr}$ and $25.5 \mathrm{mg} / \mathrm{L}$, we speculate that rheumatoid arthritis, or less likely, non-specific findings following the cervical HNP procedure which was performed 4 weeks prior to the outpatient visit,were possible diagnoses. In studies in which serial CRP and ESR levele were determined following surgical procedures without complications, the CRP level reaches a maximum level 2-3 days post-operatively $[8,9]$. Thereafter, the CRP level decreases in a biphasic pattern. On post-operative days $3-5$, the CRP decreases abruptly. Thereafter, it further decreases in a smooth manner. After 2-3 weeks post-operatively, the CRP returns to the normal value. ESR is less sensitive than CRP to surgical intervention and its level reaches a maximum level between post-operative days 5 and 6 . Thereafter, it gradually decreases $[8,9]$.

In the current case, based on the persistent elevation of the CRP and ESR levels, we assumed that these findings were a reflection of rheumatoid arthritis. Considering that there were no systemic or local signs of infection, we ruled out the possibility of septic arthritis. Although cases in which RF and FANA were negative and unilateral elbow and shoulder joint 
were invaded are untypical, rheumatoid arthritis has a great number of clinical variants. Considering that a diagnosis can be made, even in cases in which diagnostic criteria could not be met based on the guidelines proposed by The American Academy of Rheumatoid Arthritis [10], a tentative diagnosis of seronegative rheumatoid arthritis was made.

To manage this, non-steroidal anti-inflammatory agents, steroids, and other drugs, such as methotrexate and sulfasalazine, were administered to the patient during a 7-week period. Three months later, however, the patient presented with symptoms suggestive of septic arthritis. On admission, the patient underwent an aspiration of the synovial fluid and an imaging study.

Generally, in cases of septic arthritis, an acute onset over several hours to 2 days with fever and chills is typical. A prodromal phase lasting several day's, with malaise, arthralgias, and low-grade fevers, can be encountered. Pain, tenderness, erythema, heat, and soft tissue swelling in the involved joint are common. The diseases can develop by various types of organisms, most of which occur due to pyogenic bacteria. In addition, although rare, the disease can also occur due to such pathogens as M. tuberculosis, viruses, and fungi. In cases of tuberculous and fungal arthritis, the clinical manifestations and disease onset are obscure. In these cases, the diseases slowly progresses [11].

In the current case, in typical cases of septic arthritis, a diagnosis is mostly made within 1-2 weeks [12]. By contrast, the progression of the clinical course is atypically obscure and it occurs in a systematic manner. Therefore, it takes approximately 6 months for the current case to be suspected to be septic arthritis based on the initial symptoms.

The radiologic findings of tuberculous arthritis are nonspecific in the early stage. As shown in the current case, they can be easily overlooked. After several months following the onset of symptoms, there can be symptoms such as periarticular osteopenia and soft tissue swelling. On further progression of the disease, there can also be such findings as bone erosion and joint space narrowing [13]. MRI scans can visualize such findings as soft tissue swelling or joint effusion on earlier occasions [4]. Because these radiologic findings are also seen in cases of chronic septic arthritis, however, from a diagnostic perspective, they are valuable only in a limited scope $[6,13]$.

A gold standard for the diagnosis of tuberculous arthritis is to identify $M$. tuberculosis. On AFB staining of the synovial fluid, positive findings are observed in $20-25 \%$ of samples. A culture test of the synovial fluid can identify M.tuberculosis in $60-80 \%$ of samples. A tissue biopsy of synovial membrane can produce more apparent results. Although M. tuberculosis was not identified, anti-tuberculosis treatment can be initiated in cases in which there are granulomatous inflammatory findings [4]. Also, in the current case, a diagnosis of tuberculous arthritis was made based on the post-operative biopsy of the synovial membrane and AFB staining.

As shown herein, due to a lower incidence of tuberculous arthritis occurring in the elbow joint and the clinical characteristics which are obscure and gradual, a rule-out diagnosis of tuberculous arthritis could not be made in the early stage. In recent years, however, the prevalence of tuberculosis has been increasing. Moreover, although rare, tuberculous arthritis of the peripheral joint has also increased. Considering that Korea is one of the endemic areas for tuberculosis, in cases of chronic arthralgia in which there is a local swelling of the soft tissue and the ROM is gradually restricted, although rare, the possibility for tuberculous arthritis cannot be completely ruled out.

\section{References}

1. Rahman MS, Brar R, Konchwalla A, Sala MJ. Pain in the elbow: a rare presentation of skeletal tuberculosis. J Shoulder Elbow Surg 2008; 17(1): e19-21.

2. Akqun U, Erol B, Cimsit C, Karahan M. Tuberculosis of the knee joint: a case report. Acta Orthop Traumatol Turc 2008; 42: 214-8.

3. Domingo A, Nomdedeu M, Tomas X, Garcia S. Elbow tuberculosis: an unusual location and diagnostic problem. Arch Orthop Trauma Surg 2005; 125: 56-8.

4. Erden H, Baylan O, Simsek I, Dinc A, Pay S, Kocaoglu M. Delayed diagnosis of tuberculous arthritis. Jpn J Infect Dis 2005; 58: 373-5.

5. Chang DJ, Yoon DM, Kang YS, Yoon KB. Chronic back pain proven to be spinal tuberculosis. Korean J Pain 2008; 21: 74-9.

6. Hunfeld KP, Rittmeister M, Wichelhaus TA, Brade V, Enzensberger R. Two cases of chronic arthritis of the forearm due to Mycobacterium tuberculosis. Eur J Clin Microbiol Infect Dis 1998; 17: 344-8.

7. Chen WS, Wang CJ, Eng HL. Tuberculous arthritis of the elbow. Int Orthop 1997; 21: 367-70.

8. Giehl JP, Kluba T, Lebherz C. The course of inflammatory mediators after elective orthopedic interventions. Z Orthop Ihre Grenzgeb 2000; 138: 181-4.

9. Larsson S, Thelander U, Friberg S. C-reactive protein (CRP) levels after elective orthopaedic surgery. Clin Orthop Relat Res 1992; 275 : 237-42.

10. Scott DL, Steer S. The course of established rheumatoid arthritis. Best Pract Res Clin Rheumatol 2007; 21: 943-67.

11. Mohana-Borges AV, Chung CB, Resnick D. Monoarticular arthritis. Radiol Clin North Am 2004; 42: 135-49.

12. Mathews CJ, Coakley G. Septic arthritis: current diagnostic and therapeutic algorithm. Curr Opin Rheumatol 2008; 20: 457-62.

13. Asaka T, Takizawa Y, Kariya T, Nitta E, Yasuda T, Fujita M, et al. Tuberculous Tenosynovitis in the Elbow Joint. Intern Med 1996; 35: 162-5. 